International Journal of Pure and Applied Mathematics

Volume 111 No. 3 2016, 491-505

ISSN: 1311-8080 (printed version); ISSN: 1314-3395 (on-line version)

url: http://www.ijpam.eu

doi: 10.12732 /ijpam.v111i3.12

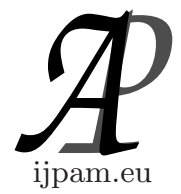

\title{
DOUBLE ABBASBANDY'S METHOD FOR SOLVING NONLINEAR EQUATIONS
}

\author{
Shah Hussain ${ }^{1}$, Shin Min Kang ${ }^{2}$, Waqas Nazeer ${ }^{3}$, Irum Sarfraz ${ }^{4}$ \\ ${ }^{1}$ Department of Mathematics \\ Minhaj University \\ Lahore, 54000, PAKISTAN \\ ${ }^{2}$ Department of Mathematics and RINS \\ Gyeongsang National University \\ Jinju, 52828, KOREA \\ ${ }^{3}$ Division of Science and Technology \\ University of Education \\ Lahore, 54000, PAKISTAN \\ ${ }^{4}$ Department of Mathematics \\ Lahore Leads University \\ Lahore 54810, PAKISTAN
}

\begin{abstract}
In this paper, we proposed a double (two-step) Abbasbandy's method for solving nonlinear equations. It is shown that the proposed iterative method has convergence of order nine and efficiency index 1.7321 . We solve some test examples to check validity and efficiency of presented algorithm.
\end{abstract}

AMS Subject Classification: 65H05, 65D32

Key Words: nonlinear equation, iterative method, Newton method, Halley's method, Househölder's method, Abbasbandy's method, Noor and Noor method, double Abbasbandy's method

Received: $\quad$ October 11, 2016

Revised: $\quad$ November 18, 2016

Published: December 19, 2016

$\S_{\text {Correspondence author }}$ (c) 2016 Academic Publications, Ltd. url: www.acadpubl.eu 


\section{Introduction}

The boundary value problems in Kinetic theory of gases, elasticity and other applied areas are mostly reduced in solving single variable nonlinear equations. Hence, the problem of approximating a solution of the nonlinear equations $f(x)=0$, is important. The numerical methods for the roots of such equations are called iterative methods [25]. Many such iterative methods for solving nonlinear equations are in literature for example $[25,24,9,17,1,26,10,22$, $23,6,4,5,7,8,11,15,16,2,3,13,14,18,19]$ and the reference therein. There are two types of iterative methods, i.e. derivative free methods [24] and, higher order iterative methods involving derivatives $[9,17,1,26,10,22,23,6,4,5,7$, $8,11,15,16,2,3,13,14,18]$. Here, we are interested in finding higher order iterative method involving derivative.

In this paper, the double (two-step) Abbasbandy's method for solving nonlinear equations. It is shown that this new algorithm has convergence or order nine and efficiency index 1.7321 .

The breakup of the paper is as follows: In the second section, we give a new iterative method (double Abbasbandy's method). In third section, we proved that convergence order of presented iterative method is at least nine. In fourth section, we compare the efficiency index of presented iterative method with some other iterative methods. In fifth section, some test examples are solved to check the fast convergence of presented iterative method. In the sixth section, polynomiography via presented the double Abbasbandy's method is given.

\section{New Iterative Method}

Consider the nonlinear algebraic equation

$$
f(x)=0 .
$$

We assume that $\alpha$ is a simple zero of Eq. (2.1) and $\gamma$ is an initial guess sufficiently close to $\alpha$. Using the Taylors series, we have

$$
\left.f(\gamma)+(x-\gamma) f^{\prime} \gamma\right)+\frac{1}{2 !}(x-\gamma)^{2} f^{\prime \prime}(\gamma)+\cdots=0
$$

If $f^{\prime}(\gamma) \neq 0$, we can evaluate the above expression (2.2) as follow

$$
f(\gamma)+(x-\gamma) f^{\prime}(\gamma)=0
$$

This formulation is used to suggest the following iterative method 
Algorithm 2.1. For a given $x_{0}$, compute the approximate solution $x_{n+1}$ by the iterative scheme

$$
x_{n+1}=x_{n}-\frac{f\left(x_{n}\right)}{f^{\prime}\left(x_{n}\right)} .
$$

This is well known the Newton's method (NM) for root-finding of nonlinear functions, which converges quadratically [25, 5].

Also from (2.2), we obtain

$$
x=\gamma-\frac{2 f(\gamma) f^{\prime}(\gamma)}{2 f^{\prime} 2(\gamma)-f(\gamma) f^{\prime \prime}(\gamma)}
$$

This formulation allows us to suggest the following iterative method for solving nonlinear equation (2.1).

Algorithm 2.2. For a given $x_{0}$, compute the approximate solution $x_{n+1}$ by the iterative scheme

$$
x_{n+1}=x_{n}-\frac{2 f\left(x_{n}\right) f^{\prime}\left(x_{n}\right)}{2 f^{\prime} 2\left(x_{n}\right)-f\left(x_{n}\right) f^{\prime \prime}\left(x_{n}\right)} .
$$

This is known as the Halley's Method (HM), which has cubic convergence $[25,9,17,6,5]$.

Algorithm 2.3. For a given $x_{0}$, compute the approximate solution $x_{n+1}$ by the iterative scheme

$$
x_{n+1}=x_{n}-\frac{f\left(x_{n}\right)}{f^{\prime}\left(x_{n}\right)}-\frac{f^{2}\left(x_{n}\right) f^{\prime \prime}\left(x_{n}\right)}{2 f^{\prime} 3\left(x_{n}\right)} .
$$

This is so-called the Househölder method (HHM), which has convergence of order three $[25,5]$.

Abbasbandy [1] improve the Newton-Raphson method by the modified Adomian decomposition method, and develop following third order iterative method.

Algorithm 2.4. For a given $x_{0}$, compute the approximate solution $x_{n+1}$ by the iterative scheme

$$
x_{n+1}=x_{n}-\frac{f\left(x_{n}\right)}{f^{\prime}\left(x_{n}\right)}-\frac{f^{2}\left(x_{n}\right) f^{\prime \prime}\left(x_{n}\right)}{2 f^{\prime} 3\left(x_{n}\right)}-\frac{f^{3}\left(x_{n}\right) f^{\prime \prime \prime}\left(x_{n}\right)}{6 f^{\prime} 4\left(x_{n}\right)} .
$$

This is so-called the Abbasbandy's method (AM) for root-finding of nonlinear functions.

Noor and Noor [21] suggested the following two-step method 
Algorithm 2.5. For a given $x_{0}$, compute the approximate solution $x_{n+1}$ by the iterative scheme

$$
\begin{aligned}
y_{n} & =x_{n}-\frac{f\left(x_{n}\right)}{f^{\prime}\left(x_{n}\right)}, \\
x_{n+1} & =y_{n}-\frac{2 f\left(y_{n}\right) f^{\prime}\left(y_{n}\right)}{2 f^{\prime} 2\left(y_{n}\right)-f\left(y_{n}\right) f^{\prime \prime}\left(y_{n}\right)} .
\end{aligned}
$$

Traub [25] considered following two-step iterative methods of convergence order three and four, respectively.

Algorithm 2.6. For a given $x_{0}$, compute the approximate solution $x_{n+1}$ by the iterative scheme

$$
\begin{array}{r}
y_{n}=x_{n}-\frac{f\left(x_{n}\right)}{f^{\prime}\left(x_{n}\right)}, \\
x_{n+1}=y_{n}-\frac{f\left(y_{n}\right)}{f^{\prime}\left(x_{n}\right)} .
\end{array}
$$

Algorithm 2.7. For a given $x_{0}$, compute the approximate solution $x_{n+1}$ by the iterative scheme

$$
\begin{aligned}
y_{n} & =x_{n}-\frac{f\left(x_{n}\right)}{f^{\prime}\left(x_{n}\right)}, \\
x_{n+1} & =y_{n}-\frac{f\left(y_{n}\right)}{f^{\prime}\left(y_{n}\right)} .
\end{aligned}
$$

For more details, see $[20,19,12]$ and the references therein.

We suggest the following new two-step Abbasbandy's method called as the double Abbasbandy's method (DAM)

Algorithm 2.8. For a given $x_{0}$, compute the approximate solution $x_{n+1}$ by the following iterative schemes:

$$
\begin{aligned}
& y_{n}=x_{n}-\frac{f\left(x_{n}\right)}{f^{\prime}\left(x_{n}\right)}-\frac{f^{2}\left(x_{n}\right) f^{\prime \prime}\left(x_{n}\right)}{2 f^{\prime}\left(x_{n}\right)}-\frac{f^{3}\left(x_{n}\right) f^{\prime \prime \prime}\left(x_{n}\right)}{6 f^{\prime} 4\left(x_{n}\right)}, \\
& x_{n+1}=y_{n}-\frac{f\left(y_{n}\right)}{f^{\prime}\left(y_{n}\right)}-\frac{f^{2}\left(y_{n}\right) f^{\prime \prime}\left(y_{n}\right)}{2 f^{\prime} 3\left(y_{n}\right)}-\frac{f^{3}\left(y_{n}\right) f^{\prime \prime \prime}\left(y_{n}\right)}{6 f^{\prime} 4\left(y_{n}\right)} .
\end{aligned}
$$




\section{Convergence Analysis}

In this section we find out the order of convergence of the double Abbasbandy's method.

Theorem 3.1. Let $\alpha$ be a simple zero of sufficiently differentiable function $f$ for an open interval $I$. If $x$ is sufficiently close to $\alpha$, then Algorithm 2.8 has 9th-order convergence.

Proof. To prove the convergence of the double Abbasbandy's method is nine, suppose that $\alpha$ is a root of the equation $f(x)=0$ and $e_{n}$ be the error at $n$-th iteration, than $e_{n}=x_{n}-\alpha$ then by using Taylor series expansion, we have

$$
\begin{gathered}
f\left(x_{n}\right)=f^{\prime}\left(x_{n}\right) e_{n}+\frac{1}{2 !} f^{\prime \prime}\left(x_{n}\right) e_{n}^{2}+\frac{1}{3 !} f^{\prime \prime \prime}\left(x_{n}\right) e_{n}^{3}+\frac{1}{4 !} f^{(i v)}\left(x_{n}\right) e_{n}^{4} \\
+\frac{1}{5 !} f^{(v)}\left(x_{n}\right) e_{n}^{5}+\frac{1}{6 !} f^{(v i)}\left(x_{n}\right) e_{n}^{6}+O\left(e_{n}^{7}\right) \\
\begin{array}{c}
f\left(x_{n}\right)=f^{\prime}(\alpha)\left[e_{n}+c_{2} e_{n}^{2}+c_{3} e_{n}^{3}+c_{4} e_{n}^{4}+c_{5} e_{n}^{5}\right. \\
\left.+c_{6} e_{n}^{6}+c_{7} e_{n}^{7}+O\left(e_{n}^{8}\right)\right] \\
f^{\prime}\left(x_{n}\right)=f^{\prime}(\alpha)\left[1+2 c_{2} e_{n}+3 c_{3} e_{n}^{2}+4 c_{4} e_{n}^{3}+5 c_{5} e_{n}^{4}\right. \\
\left.+6 c_{6} e_{n}^{5}+7 c_{7} e_{n}^{6}+O\left(e_{n}^{7}\right)\right] \\
\left.+42 c_{7} e_{n}^{5}+56 c_{8} e_{n}^{6}+72 c_{9} e_{n}^{7}+O\left(e_{n}^{8}\right)\right] \\
f^{\prime \prime}\left(x_{n}\right)=f^{\prime 2}(\alpha)\left[2 c_{2}+6 c_{3} e+12 c_{4} e_{n}^{2}+20 c_{5} e_{n}^{3}+30 c_{6} e_{n}^{4}\right. \\
f^{\prime \prime \prime}\left(x_{n}\right)=f^{\prime 3}(\alpha)\left[6 c_{3}+24 c_{4} e_{n}+60 c_{5} e_{n}^{2}+120 c_{6} e_{n}^{3}+210 c_{7} e_{n}^{4}\right. \\
\left.+336 c_{8} e_{n}^{5}+504 c_{9} e_{n}^{6}+O\left(e_{n}^{7}\right)\right]
\end{array}
\end{gathered}
$$

where

$$
c_{n}=\frac{1}{n !} \frac{f^{(n)}(\alpha)}{f^{\prime}(\alpha)} .
$$


By using (3.1)-(3.4) in (2.4), we have

$$
\begin{aligned}
y_{n}= & f^{\prime}(\alpha)\left[\alpha+\left(-2 c_{3}+2 c_{2}^{2}\right) e_{n}^{3}+\left(17 c_{2} c_{3}-9 c_{2}^{3}-7 c_{4}\right) e_{n}^{4}\right. \\
+ & \left(-16 c_{5}+44 c_{2} c_{4}+24 c_{3}^{2}-82 c_{3} c_{2}^{2}+30 c_{2}^{4}\right) e_{n}^{5} \\
+ & \left(-30 c_{6}+90 c_{2} c_{5}+104 c_{4} c_{3}-188 c_{4} c_{2}^{2}-202 c_{2} c_{3}^{2}+314 c_{3} c_{2}^{3}\right. \\
& \left.-88 c_{2}^{5}\right) e_{n}^{6}+\left(-50 c_{7}+160 c_{2} c_{6}+194 c_{5} c_{3}-364 c_{5} c_{2}^{2}+100 c_{4}^{2}\right. \\
& \left.+672 c_{4} c_{2}^{3}+1074 c_{3}^{2} c_{2}^{2}-1056 c_{3} c_{2}^{4}-820 c_{2} c_{4} c_{3}-150 c_{3}^{3}+240 c_{2}^{6}\right) e_{n}^{7} \\
+ & \left(-77 c_{8}-1478 c_{2} c_{5} c_{3}+4185 c_{4} c_{2}^{2} c_{3}+259 c_{2} c_{7}-629 c_{2}^{2} c_{6}\right. \\
& +1257 c_{5} c_{2}^{3}-757 c_{2} c_{4}^{2}-2160 c_{4} c_{2}^{4}-4578 c_{3}^{2} c_{2}^{3}+3264 c_{3} c_{2}^{5} \\
& \left.+1515 c_{2} c_{3}^{3}-849 c_{4} c_{3}^{2}+327 c_{3} c_{6}+345 c_{4} c_{5}-624 c_{2}^{7}\right) e_{n}^{8} \\
+ & \left(-112 c_{9}+7374 c_{5} c_{3} c_{2}^{2}-2444 c_{2} c_{3} c_{6}-2552 c_{2} c_{4} c_{5}+8340 c_{2} c_{4} c_{3}^{2}\right. \\
& -17336 c_{4} c_{3} c_{2}^{3}+512 c_{3} c_{7}-1456 c_{5} c_{3}^{2}-1502 c_{3} c_{4}^{2}-9348 c_{3}^{3} c_{2}^{2} \\
& +17060 c_{3}^{2} c_{2}^{4}-9504 c_{3} c_{2}^{6}+6464 c_{4} c_{2}^{5}+3762 c_{4}^{2} c_{2}^{2}+552 c_{4} c_{6} \\
& +764 c_{3}^{4}-3944 c_{5} c_{2}^{4}+392 c_{2} c_{8}+280 c_{5}^{2}-1002 c_{7} c_{2}^{2}+2132 c_{6} c_{2}^{3} \\
& \left.\left.+1568 c_{2}^{8}\right) e_{n}^{9}+O\left(e_{n}^{10}\right)\right],
\end{aligned}
$$

$$
\begin{aligned}
f\left(y_{n}\right)=f^{\prime} & (\alpha)\left[\left(-2 c_{3}+2 c_{2}^{2}\right) e_{n}^{3}+\left(17 c_{2} c_{3}-9 c_{2}^{3}-7 c_{4}\right) e_{n}^{4}\right. \\
+ & \left(-16 c_{5}+44 c_{2} c_{4}+24 c_{3}^{2}-82 c_{3} c_{2}^{2}+30 c_{2}^{4}\right) e_{n}^{5}+\left(-30 c_{6}\right. \\
& \left.+90 c_{2} c_{5}+104 c_{4} c_{3}-188 c_{4} c_{2}^{2}-198 c_{2} c_{3}^{2}+306 c_{3} c_{2}^{3}-84 c_{2}^{5}\right) e_{n}^{6} \\
+ & \left(-50 c_{7}+160 c_{2} c_{6}+194 c_{5} c_{3}-364 c_{5} c_{2}^{2}+100 c_{4}^{2}+644 c_{4} c_{2}^{3}\right. \\
& \left.+1006 c_{3}^{2} c_{2}^{2}-952 c_{3} c_{2}^{4}-792 c_{2} c_{4} c_{3}-150 c_{3}^{3}+204 c_{2}^{6}\right) e_{n}^{7} \\
+ & \left(-1414 c_{2} c_{5} c_{3}+3771 c_{4} c_{2}^{2} c_{3}+1419 c_{2} c_{3}^{3}-3865 c_{3}^{2} c_{2}^{3}\right. \\
& +2510 c_{3} c_{2}^{5}+1193 c_{5} c_{2}^{3}-1858 c_{4} c_{2}^{4}-423 c_{2}^{7}-708 c_{2} c_{4}^{2}-77 c_{8} \\
& \left.+259 c_{2} c_{7}-629 c_{2}^{2} c_{6}-849 c_{4} c_{3}^{2}+327 c_{3} c_{6}+345 c_{4} c_{5}\right) e_{n}^{8} \\
+ & -112 c_{9}+6470 c_{5} c_{3} c_{2}^{2}-2324 c_{2} c_{3} c_{6}-2328 c_{2} c_{4} c_{5} \\
& +7588 c_{2} c_{4} c_{3}^{2}-13524 c_{4} c_{3} c_{2}^{3}+512 c_{3} c_{7}-1456 c_{5} c_{3}^{2} \\
& -1502 c_{3} c_{4}^{2}-7700 c_{3}^{3} c_{2}^{2}+11752 c_{3}^{2} c_{2}^{4}-5392 c_{3} c_{2}^{6}+4500 c_{4} c_{2}^{5} \\
& +3146 c_{4}^{2} c_{2}^{2}+552 c_{4} c_{6}+756 c_{3}^{4}-3296 c_{5} c_{2}^{4}+392 c_{2} c_{8}+280 c_{5}^{2} \\
& \left.\left.-1002 c_{7} c_{2}^{2}+2012 c_{6} c_{2}^{3}+676 c_{2}^{8}\right) e_{n}^{9}+O\left(e_{n}^{10}\right)\right]
\end{aligned}
$$




$$
\begin{aligned}
f^{\prime}\left(y_{n}\right)=f^{\prime} & (\alpha)\left[1+\left(-4 c_{2} c_{3}+4 c_{2}^{3}\right) e_{n}^{3}+\left(34 c_{3} c_{2}^{2}-18 c_{2}^{4}-14 c_{2} c_{4}\right) e_{n}^{4}\right. \\
+ & \left(-32 c_{2} c_{5}+88 c_{4} c_{2}^{2}+48 c_{2} c_{3}^{2}-164 c_{3} c_{2}^{3}+60 c_{2}^{5}\right) e_{n}^{5} \\
+ & \left(-60 c_{2} c_{6}+180 c_{5} c_{2}^{2}+208 c_{4} c_{2} c_{3}-376 c_{4} c_{2}^{3}-428 c_{3}^{2} c_{2}^{2}\right. \\
& \left.+640 c_{3} c_{2}^{4}-176 c_{2}^{6}+12 c_{3}^{3}\right) e_{n}^{6}+\left(-100 c_{2} c_{7}+320 c_{2}^{2} c_{6}\right. \\
& +388 c_{2} c_{5} c_{3}-728 c_{5} c_{2}^{3}+200 c_{2} c_{4}^{2}+1344 c_{4} c_{2}^{4}+2460 c_{3}^{2} c_{2}^{3} \\
& \left.-2220 c_{3} c_{2}^{5}-1724 c_{4} c_{2}^{2} c_{3}-504 c_{2} c_{3}^{3}+480 c_{2}^{7}+84 c_{4} c_{3}^{2}\right) e_{n}^{7} \\
+ & \left(192 c_{5} c_{3}^{2}-2940 c_{4} c_{2} c_{3}^{2}-288 c_{3}^{4}+5169 c_{3}^{3} c_{2}^{2}-11418 c_{3}^{2} c_{2}^{4}\right. \\
& -3148 c_{3} c_{5} c_{2}^{2}+9276 c_{3} c_{4} c_{2}^{3}+7131 c_{3} c_{2}^{6}+147 c_{3} c_{4}^{2}-154 c_{2} c_{8} \\
& +518 c_{7} c_{2}^{2}-1258 c_{6} c_{2}^{3}+2514 c_{5} c_{2}^{4}-1514 c_{4}^{2} c_{2}^{2}-4320 c_{4} c_{2}^{5} \\
& \left.+654 c_{3} c_{2} c_{6}+690 c_{4} c_{2} c_{5}-1248 c_{2}^{8}\right) e_{n}^{8}+\left(4264 c_{6} c_{2}^{4}\right. \\
& +1024 c_{2} c_{3} c_{7}+12960 c_{4} c_{2}^{6}-7888 c_{5} c_{2}^{5}+360 c_{3}^{2} c_{6}+6400 c_{2} c_{3}^{4} \\
& -34548 c_{3}^{3} c_{2}^{3}+16692 c_{5} c_{3} c_{2}^{3}-5248 c_{3} c_{2}^{2} c_{6}+46432 c_{3}^{2} c_{2}^{5} \\
& -21684 c_{3} c_{2}^{7}-224 c_{2} c_{9}+7524 c_{4}^{2} c_{2}^{3}-2288 c_{4} c_{3}^{3}+1104 c_{4} c_{2} c_{6} \\
& -4852 c_{4}^{2} c_{2} c_{3}-40660 c_{4} c_{3} c_{2}^{4}+672 c_{4} c_{5} c_{3}-5104 c_{4} c_{5} c_{2}^{2} \\
& -5624 c_{5} c_{2} c_{3}^{2}+28212 c_{4} c_{3}^{2} c_{2}^{2}+784 c_{2}^{2} c_{8}+560 c_{2} c_{5}^{2} \\
& \left.\left.-2004 c_{7} c_{2}^{3}+3136 c_{2}^{9}\right) e_{n}^{9}+O\left(e_{n}^{10}\right)\right],
\end{aligned}
$$




$$
\begin{aligned}
f^{\prime \prime}\left(y_{n}\right)=f^{\prime 2} & (\alpha)\left[2 c_{2}+\left(-12 c_{3}^{2}+12 c_{3} c_{2}^{2}\right) e_{n}^{3}+\left(102 c_{2} c_{3}^{2}-54 c_{3} c_{2}^{3}\right.\right. \\
& \left.-42 c_{3} c_{4}\right) e_{n}^{4}+\left(-96 c_{3} c_{5}+264 c_{4} c_{2} c_{3}+144 c_{3}^{3}-492 c_{3}^{2} c_{2}^{2}\right. \\
& \left.+180 c_{3} c_{2}^{4}\right) e_{n}^{5}+\left(-180 c_{3} c_{6}+540 c_{2} c_{5} c_{3}+672 c_{4} c_{3}^{2}\right. \\
& \left.-1224 c_{4} c_{2}^{2} c 3-1212 c_{2} c_{3}^{3}+1884 c_{3}^{2} c_{2}^{3}-528 c_{3} c_{2}^{5}+48 c_{4} c_{2}^{4}\right) e_{n}^{6} \\
+ & \left(-300 c_{3} c_{7}+960 c_{3} c_{2} c_{6}+1164 c_{5} c_{3}^{2}-2184 c_{3} c_{5} c_{2}^{2}+936 c_{3} c_{4}^{2}\right. \\
& +5280 c_{3} c_{4} c_{2}^{3}+6444 c_{3}^{3} c_{2}^{2}-6336 c_{3}^{2} c_{2}^{4}-5736 c_{4} c_{2} c 3^{2} \\
& \left.-900 c_{3}^{4}+1440 c_{3} c_{2}^{6}-432 c 4 c_{2}^{5}-336 c_{4}^{2} c_{2}^{2}\right) e_{n}^{7} \\
+ & -2838 c_{4} c_{5} c_{3}-9510 c_{4}^{2} c_{2} c_{3}-6246 c_{4} c_{3}^{3}+33666 c_{4} c_{3}^{2} c_{2}^{2} \\
& -22008 c_{4} c_{3} c_{2}^{4}-768 c_{4} c_{5} c_{2}^{2}+3624 c_{4}^{2} c_{2}^{3}+2412 c_{4} c_{2}^{6}+588 c_{4}^{3} \\
& -462 c_{3} c_{8}-8868 c_{5} c_{2} c_{3}^{2}+1554 c_{2} c_{3} c_{7}-3774 c_{3} c_{2}^{2} c_{6} \\
& +7542 c_{5} c_{3} c_{2}^{3}-27468 c_{3}^{3} c_{2}^{3}+19584 c_{3}^{2} c_{2}^{5}+9090 c_{2} c_{3}^{4} \\
& \left.+1962 c_{3}^{2} c_{6}-3744 c_{3} c_{2}^{7}\right) e_{n}^{8}+\left(4584 c_{3}^{5}-167424 c_{4} c_{3}^{2} c_{2}^{3}\right. \\
& -6012 c_{3} c_{7} c_{2}^{2}+2352 c_{2} c_{3} c_{8}+69528 c_{2} c_{4} c_{3}^{3}+68316 c_{4}^{2} c_{2}^{2} c_{3} \\
& +88032 c_{3} c_{4} c_{2}^{5}+7776 c_{4} c_{5} c_{2}^{3}-1440 c_{4} c_{2}^{2} c_{6}+44724 c_{5} c_{3}^{2} c_{2}^{2} \\
& -14664 c_{2} c_{3}^{2} c_{6}+4752 c_{3} c_{4} c_{6}+12792 c_{3} c_{6} c_{2}^{3}-24144 c_{3} c_{5} c_{2}^{4} \\
& -18036 c_{4}^{2} c_{3}^{2}-23568 c_{4}^{2} c_{2}^{4}-10704 c_{4} c_{2}^{7}+2688 c_{4}^{2} c_{5} \\
& -7392 c_{2} c_{4}^{3}-672 c_{3} c_{9}+3072 c_{3}^{2} c_{7}-8896 c_{5} c_{3}^{3}-56088 c_{3}^{4} c_{2}^{2} \\
& +102360 c_{3}^{3} c_{2}^{4}-57024 c_{3}^{2} c_{2}^{6}+1680 c_{3} c_{5}^{2}+9408 c_{3} c_{2}^{8}+160 c_{5} c_{2}^{6} \\
& \left.\left.-26160 c_{4} c_{2} c_{5} c_{3}\right) e_{n}^{9}+O\left(e_{n}^{10}\right)\right] \\
& -14
\end{aligned}
$$




$$
\begin{aligned}
& f^{\prime \prime \prime}\left(y_{n}\right)=f^{\prime 3}(\alpha)\left[6 c_{3}+\left(-48 c_{4} c_{3}+48 c_{4} c_{2}^{2}\right) e_{n}^{3}+\left(408 c_{4} c_{2} c_{3}-216 c_{4} c_{2}^{3}\right.\right. \\
& \left.-168 c_{4}^{2}\right) e_{n}^{4}+\left(-384 c_{4} c_{5}+1056 c_{2} c_{4}^{2}+576 c_{4} c_{3}^{2}-1968 c_{4} c_{2}^{2} c_{3}\right. \\
& \left.+720 c_{4} c_{2}^{4}\right) e_{n}^{5}+\left(-720 c_{4} c_{6}+2160 c_{4} c_{2} c_{5}+2496 c_{3} c_{4}^{2}\right. \\
& -4512 c_{4}^{2} c_{2}^{2}-4848 c_{4} c_{2} c_{3}^{2}+7536 c_{3} c_{4} c_{2}^{3}-2112 c_{4} c_{2}^{5}+240 c_{3}^{2} c_{5} \\
& \left.-480 c_{3} c_{5} c_{2}^{2}+240 c_{5} c_{2}^{4}\right) e_{n}^{6}+\left(-1200 c_{4} c_{7}+3840 c_{4} c_{2} c_{6}\right. \\
& +6336 c_{4} c_{5} c_{3}-10416 c_{4} c_{5} c_{2}^{2}+2400 c_{4}^{3}+16128 c_{4}^{2} c_{2}^{3} \\
& +25776 c_{4} c_{3}^{2} c_{2}^{2}-25344 c_{4} c_{3} c_{2}^{4}-19680 c_{4}^{2} c_{2} c_{3}-3600 c_{4} c_{3}^{3} \\
& \left.+5760 c_{4} c_{2}^{6}-4080 c_{5} c_{2} c_{3}^{2}+6240 c_{5} c_{3} c_{2}^{3}-2160 c_{5} c_{2}^{5}\right) e_{n}^{7} \\
& +\left(3840 c_{3} c_{5}^{2}-60312 c_{4} c_{2} c_{5} c_{3}-5760 c_{5} c_{3}^{3}+42780 c_{3}^{2} c_{5} c_{2}^{2}\right. \\
& -45240 c_{3} c_{5} c_{2}^{4}-3840 c_{5}^{2} c_{2}^{2}+48288 c_{4} c_{5} c_{2}^{3}+12060 c_{5} c_{2}^{6} \\
& +11220 c_{4}^{2} c_{5}-1848 c_{4} c_{8}+100440 c_{4}^{2} c_{2}^{2} c_{3}+6216 c_{4} c_{2} c_{7} \\
& -15096 c_{4} c_{2}^{2} c_{6}-18168 c_{2} c_{4}^{3}-51840 c_{4}^{2} c_{2}^{4}-109872 c_{4} c_{3}^{2} c_{2}^{3} \\
& +78336 c_{4} c_{3} c_{2}^{5}+36360 c_{4} c_{2} c_{3}^{3}-20376 c_{4}^{2} c_{3}^{2}+7848 c_{4} c_{3} c_{6} \\
& \left.-14976 c_{4} c_{2}^{7}\right) e_{n}^{8}+\left(2880 c_{6} c_{3}^{2} c_{2}^{2}-80064 c_{5} c_{4} c_{3}^{2}\right. \\
& +409440 c_{4} c_{3}^{2} c_{2}^{4}+200160 c_{4}^{2} c_{2} c_{3}^{2}+51168 c_{4} c_{6} c_{2}^{3}+155136 c_{4}^{2} c_{2}^{5} \\
& -36048 c_{3} c_{4}^{3}-2688 c_{4} c_{9}-54240 c_{2} c_{5}^{2} c_{3}-98208 c_{5} c_{2} c_{4}^{2} \\
& +97440 c_{5} c_{2} c_{3}^{3}-224352 c_{4} c_{3}^{3} c_{2}^{2}+38880 c_{5}^{2} c_{2}^{3}+18336 c_{4} c_{3}^{4} \\
& +37632 c_{4} c_{2}^{8}-960 c_{6} c_{3}^{3}+960 c_{6} c_{2}^{6}+13248 c_{4}^{2} c_{6}+20160 c_{4} c_{5}^{2} \\
& +405696 c_{5} c_{4} c_{2}^{2} c_{3}-58656 c_{4} c_{3} c_{2} c_{6}+9408 c_{4} c_{2} c_{8} \\
& +12288 c_{4} c_{3} c_{7}+90288 c_{4}^{3} c_{2}^{2}+7200 c_{5} c_{3} c_{6}-53520 c_{5} c_{2}^{7} \\
& +246240 c_{5} c_{3} c_{2}^{5}-228096 c_{4} c_{3} c_{2}^{6}-7200 c_{5} c_{2}^{2} c_{6}-212496 c_{5} c_{4} c_{2}^{4} \\
& -416064 c_{3} c_{4}^{2} c_{2}^{3}-317040 c_{5} c_{3}^{2} c_{2}^{3}-2880 c_{6} c_{3} c_{2}^{4} \\
& \left.\left.-24048 c_{4} c_{7} c_{2}^{2}\right) e_{n}^{9}+O\left(e_{n}^{10}\right)\right] \text {, }
\end{aligned}
$$

hence

$$
x_{n+1}=\alpha+\left(-64 c_{3}^{3} c_{2}^{2}+96 c_{3}^{2} c_{2}^{4}-64 c_{3} c_{2}^{6}+16 c_{3}^{4}+16 c_{2}^{8}\right) e_{n}^{9}+O\left(e_{n}^{10}\right),
$$

which implies that

$$
e_{n+1}=\left(-64 c_{3}^{3} c_{2}^{2}+96 c_{3}^{2} c_{2}^{4}-64 c_{3} c_{2}^{6}+16 c_{3}^{4}+16 c_{2}^{8}\right) e_{n}^{9}+O\left(e_{n}^{10}\right),
$$

which shows that Algorithm 2.8 has 9th-order convergence. 


\section{Comparisons of Efficiency Index}

We use "efficiency index" knowing about the performance of different iterative methods, which depends upon the order of convergence and number of functional evaluations of the iterative method, where $m$ denote the order of convergence and $N_{f}$ denote the number of functional evaluations of an iterative method, then the efficiency index E.I is defined as:

$$
E . I=m^{\frac{1}{N_{f}}} .
$$

On this basis, the Newton's method has number of functional evaluations two and order of convergence quadratic so having efficiency of $2^{\frac{1}{2}} \approx 1.4142$, the Abbasbandy's method have an efficiency of $3^{\frac{1}{4}} \approx 1.3161$. with order of convergence is cubic.

We calculate the efficiency index of our new developed double Abbasbandy's method as follows: The double Abbasbandy's method need one evaluation of the function and three of its first, second and third derivatives. So the number of functional evaluations of this method is four, thta is,

$$
N_{f}=4 \text {. }
$$

In Theorem 3.1, we have proved that the order of convergence of our double Abbasbandy's method is nine, that is,

$$
m=9 .
$$

So the efficiency index of the double Abbasbandy's method is:

$$
E . I=9^{\frac{1}{4}} \approx 1.7321 .
$$

\section{Numerical Examples}

We present some examples to illustrate the efficiency of the developed double Abbasbandy's method (DAM) in this paper. We compare the Newton method (NM), the Halley's method (HM), the Househölder's method (HHM), the Abbasbandy's method (AM), the Noor and Noor method (NNM) and our new double Abbasbandy's method (DAM) (Algorithm 2.8) introduced in this 
present paper. We used $\varepsilon=10^{-15}$. The following stopping criteria is used for computer programs:

$$
\begin{aligned}
& f_{1}(x)=(x-1)^{3}-1, \\
& f_{2}(x)=\cos x-x, \\
& f_{3}(x)=x^{3}+x^{2}-2, \\
& f_{4}(x)=e^{x}-4 x^{2}, \\
& f_{5}(x)=x^{3}-10 .
\end{aligned}
$$

Table 1. Comparison of NM, HM, HHM, AM, NNM and DAM

$$
\left(f_{1}(x)=(x-1)^{3}-1, x_{0}=1.5\right)
$$

\begin{tabular}{|c|c|c|c|c|}
\hline Method & $N$ & $N_{f}$ & $\left|f\left(x_{n+1}\right)\right|$ & $x_{n+1}$ \\
\hline NM & 7 & 14 & $1.091232 e-22$ & \\
\hline HM & 4 & 12 & $6.390950 e-24$ & 2.000000000000000000000000000000 \\
\hline HHM & 52 & 156 & $7.662031 e-28$ & \\
\hline AM & 4 & 16 & $7.139947 e-32$ & \\
\hline NNM & 4 & 12 & $6.390950 e-24$ & \\
\hline DAM & 2 & 8 & $7.139947 e-32$ & \\
\hline
\end{tabular}

Table 2-1. Comparison of NM, HM, HHM, AM, NNM and DAM

$$
\left(f_{2}(x)=\cos x-x, x_{0}=0.75\right)
$$

\begin{tabular}{|c|c|c|c|c|}
\hline Method & $N$ & $N_{f}$ & $\left|f\left(x_{n+1}\right)\right|$ & $x_{n+1}$ \\
\hline NM & 3 & 6 & $8.239929 e-21$ & \\
\hline HM & 2 & 6 & $6.503146 e-22$ & 0.739085133215160641655312087674 \\
\hline HHM & 2 & 6 & $2.585651 e-21$ & \\
\hline AM & 2 & 8 & $1.027784 e-20$ & \\
\hline NNM & 3 & 9 & $6.503146 e-22$ & \\
\hline DAM & 1 & 4 & $1.027784 e-20$ & \\
\hline
\end{tabular}

Table 2-2. Comparison of NM, HM, HHM, AM, NNM and DAM

$$
\left(f_{2}(x)=\cos x-x, x_{0}=0\right)
$$

\begin{tabular}{|c|c|c|c|c|}
\hline Method & $N$ & $N_{f}$ & $\left|f\left(x_{n+1}\right)\right|$ & $x_{n+1}$ \\
\hline NM & 5 & 10 & $1.069528 e-20$ & \\
\hline HM & 4 & 12 & $2.709552 e-43$ & 0.739085133215160641655312087674 \\
\hline HHM & 4 & 12 & $4.166298 e-26$ & \\
\hline AM & 4 & 16 & $1.529541 e-24$ & \\
\hline NNM & 4 & 12 & $2.709552 e-43$ & \\
\hline DAM & 2 & 8 & $1.529541 e-24$ & \\
\hline
\end{tabular}


Table 3. Comparison of NM, HM, HHM, AM, NNM and DAM $\left(f_{3}(x)=x^{3}+x^{2}-2, x_{0}=1.6\right)$

\begin{tabular}{|c|c|c|c|c|}
\hline Method & $N$ & $N_{f}$ & $\left|f\left(x_{n+1}\right)\right|$ & $x_{n+1}$ \\
\hline NM & 6 & 12 & $8.874553 e-29$ & \\
\hline HM & 4 & 12 & $4.144560 e-41$ & 1.000000000000000000000000000000 \\
\hline HHM & 4 & 12 & $9.417942 e-31$ & \\
\hline AM & 4 & 16 & $5.905712 e-33$ & \\
\hline NNM & 4 & 12 & $4.144560 e-41$ & \\
\hline DAM & 2 & 8 & $5.905712 e-33$ & \\
\hline
\end{tabular}

Table 4-1. Comparison of NM, HM, HHM, AM, NNM and DAM

$$
\left(f_{4}(x)=e^{x}-4 x^{2}, x_{0}=1.5\right)
$$

\begin{tabular}{|c|c|c|c|c|}
\hline Method & $N$ & $N_{f}$ & $\left|f\left(x_{n+1}\right)\right|$ & $x_{n+1}$ \\
\hline NM & 6 & 12 & $1.031370 e-28$ & \\
\hline HM & 4 & 12 & $2.000988 e-31$ & 0.714805912362777806137622208112 \\
\hline HHM & 4 & 12 & $1.053161 e-26$ & \\
\hline AM & 4 & 16 & $2.687756 e-24$ & \\
\hline NNM & 4 & 12 & $2.000988 e-31$ & \\
\hline DAM & 2 & 8 & $2.687756 e-24$ & \\
\hline
\end{tabular}

Table 4-2. Comparison of NM, HM, HHM, AM, NNM and DAM

$$
\left(f_{4}(x)=e^{x}-4 x^{2}, x_{0}=1.45\right)
$$

\begin{tabular}{|c|c|c|c|c|}
\hline Method & $N$ & $N_{f}$ & $\left|f\left(x_{n+1}\right)\right|$ & $x_{n+1}$ \\
\hline NM & 5 & 10 & $7.187888 e-15$ & \\
\hline HM & 4 & 12 & $6.440701 e-33$ & 0.714805912362777806137622208112 \\
\hline HHM & 4 & 12 & $6.156572 e-28$ & \\
\hline AM & 4 & 16 & $1.138729 e-25$ & \\
\hline NNM & 4 & 12 & $6.440701 e-33$ & \\
\hline DAM & 2 & 8 & $1.138729 e-25$ & \\
\hline
\end{tabular}

Table 5. Comparison of NM, HM, HHM, AM, NNM and DAM

$$
\left(f_{5}(x)=x^{3}-10, x_{0}=1\right)
$$

\begin{tabular}{|c|c|c|c|c|}
\hline Method & $N$ & $N_{f}$ & $\left|f\left(x_{n+1}\right)\right|$ & $x_{n+1}$ \\
\hline NM & 7 & 14 & $1.957750 e-17$ & \\
\hline HM & 4 & 12 & $4.423005 e-20$ & 2.154434690031883721759293566520 \\
\hline HHM & 17 & 51 & $1.278188 e-33$ & \\
\hline AM & 5 & 20 & $9.499846 e-20$ & \\
\hline NNM & 4 & 12 & $4.423005 e-20$ & \\
\hline DAM & 3 & 12 & $1.270124 e-60$ & \\
\hline
\end{tabular}


Tables 1-5 shows the numerical comparisons of the Newton's method (NM), the Halley's method (HM), the Househölder's method (HHM), the Abbasbandy's method (AM), the Noor and Noor's method (NNM) and the new double Abbasbanday's method (DAM)(Algorithm 2.8). The columns represent the number of iterations $N$ and the number of functions or derivatives evaluations $N_{f}$ required to meet the stopping criteria, and the magnitude $|f(x)|$ of $f(x)$ at the final estimate $x_{n}$.

\section{Conclusions}

A new double Abbasbandy's method (DAM) for solving nonlinear functions has been obtained. We can concluded from Tables 1-5 that:

1. The efficiency index of DAM is 1.7321 , which is higher than many existing methods.

2.The convergence order of DAM is nine, which is higher than many existing methods.

3. From Tables 1-5, it can be observed that our presented iterative method (DAM) perform better than the Newton's method, the Halley's method, the Househölder's method, the Abbasbandy's method and the Noor and Noor's method.

\section{References}

[1] S. Abbasbandy, Improving Newton-Raphson method for nonlinear equa-tions by modified Adomian decomposition method, Appl. Math. Comput., 145 (2003), 887-893, doi: 10.1016/S0096-3003(03)00282-0.

[2] A. Ali, M.S. Ahmad, W. Nazeer, M. Tanveer, New modified two-step jungck iterative method for solving nonlinear functional equations, Sci. Int. (Lahore), 27 (2015), 29592963.

[3] A. Ali, Q. Mehmood, M. Tanveer, A. Aslam, W. Nazeer, Modified new third-order iterative method for non-linear equations, Sci. Int. (Lahore), 27 (2015), 1741-1744.

[4] E. Babolian, J. Biazar, Solution of nonlinear equations by modified Adomian decomposition method, Appl. Math. Comput., 132 (2002), 167-172, doi: 10.1016/S00963003(01)00184-9.

[5] R.L. Burden, J.D. Faires, Numerical Analysis (Sixth ed.), Brooks/Cole Publishing Co., California, 1998.

[6] D. Chen, I.K. Argyros, Q.S. Qian, A note on the Halley method in Banach spaces, Appl. Math. Comput., 58 (1993), 215-224, doi: 10.1016/0096-3003(93)90137-4. 
[7] M. Frontini, E. Sormani, Some variant of Newton's method with third-order convergence, Appl. Math. Comput., 140 (2003), 419-426, doi: 10.1016/S0096-3003(02)00238-2.

[8] A. Golbabai, M. Javidi, A third-order Newton type method for nonlinear equations based on modified homotopy perturbation method, Appl. Math. Comput., 191 (2007), 199-205, doi: 10.1016/j.amc.2007.02.079.

[9] E. Halley, A new exact and easy method for finding the roots of equations generally and without any previous reduction, Phil. Roy. Soc. London, 18 (1964) 136-147.

[10] H. Homerier, A modified Newton's Method for root finding with cubic convergence, $J$. Comput. Appl. Math., 157 (2003), 227-230, doi: 10.1016/S0377-0427(03)00391-1.

[11] P. Jarratt, Some efficient fourth order multipoint methods for solving equations, BIT, 9 (1969), 119-124, doi: 10.1007/BF01933248.

[12] S.M. Kang, A. Naseem, W. Nazeer and A. Jan, Modification of Abbasbandy's method and polynomigraphy, Int. J. Math. Anal., 10 (2016), 1197-1210, doi: 10.12988/ijma.2016.6898.

[13] S.M. Kang, W. Nazeer, A. Rafiq, C.Y. Jung, A new third order iterative method for scalar nonlinear equations, Int. J. Math. Anal., 8 (2014), 2141-2150, doi: 10.12988/ijma.2014.48236.

[14] S.M. Kang, W. Nazeer, M. Tanveer, Q. Mehmood, K. Rehman, Improvements in NewtonRapshon method for nonlinear equations using modified Adomian decomposition method, Int. J. Math. Anal., 9 (2015), 1919-1928, doi: 10.12988/ijma.2015.54124.

[15] J. Kuo, The improvements of modified Newton's method, Appl. Math. Comput., 189 (2007), 602-609, doi: 10.1016/j.amc.2006.11.115.

[16] T.J. McDougall, S.J. Wotherspoon, A simple modification of Newton's method to achieve convergence of order $1+\sqrt{2}$, Appl. Math. Lett., 29 (2014), 20-25, doi: 10.1016/j.aml.2013.10.008.

[17] A. Melman, Geometry and convergence of Halley's method, SIAM Rev., 39 (1997), 728735.

[18] W. Nazeer, S.M. Kang, M. Tanveer, Modified Abbasbandy's method for solving nonlinear functions with convergence of order six, Int. J. Math. Anal., 9 (2015), 2011-2019, doi: 10.12988/ijma.2015.56166.

[19] W. Nazeer, M. Tanveer, S.M. Kang, A. Naseem, A new Householder's method free from second derivatives for solving nonlinear equations and polynomiography, J. Nonlinear Sci. Appl., 9 (2016), 998-1007.

[20] W. Nazeer, M. Tanveer, K. Rehman and S.M. Kang, Modified new sixth-order fixed point iterative methods for solving nonlinear functional equations, Int. J. Pure Appl. Math., 109 (2016), 223-232, doi: 10.12732/ijpam.v109i2.5.

[21] K.I. Noor, M.A. Noor, Predictor-corrector Halley method for nonlinear equations, Appl. Math. Comput., 188 (2007), 1587-1591, doi: 10.1016/j.amc.11.023.

[22] M.A. Noor, Some iterative methods for solving nonlinear equations using homotopy perturbation method, Int. J. Comput. Math., 87 (2010), 141-149, doi: 10.1080/00207160801969513.

[23] M.A. Noor, K.I. Noor, Three-step iterative methods for nonlinear equations, Appl. Math. Comput., 183 (2006), 322-327, doi: 10.1016/j.amc.2006.05.055. 
[24] F. Soleymani, Optimal fourth-order iterative methods free from derivative, Miskolc Math. Notes, 12 (2011), 255-264.

[25] J.F. Traub, Iterative Methods for the Solution of Equations, AMS Chelsea Publishing, New York, 1982.

[26] S. Weerakoon, T.G.I. Fernando, A variant of Newton's method with accelerated third-order convergence, Appl. Math. Lett., 13 (2000), 87-93, doi: 10.1016/S08939659(00)00100-2. 
